\title{
Use of biological motion based cues and ecological sounds in the neurorehabilitation of apraxia.
}

\author{
Marta Bieńkiewicz ${ }^{1}$, Georg Goldenberg ${ }^{2}$, José M. Cogollor ${ }^{3}$, Manuel Ferre ${ }^{3}$, Charmayne Hughes ${ }^{1}$ and \\ Joachim Hermsdörfer ${ }^{1}$ \\ ${ }^{1}$ Technische Universität München, Lehrstuhl für Bewegungswissenschaft, Uptown München-Campus D \\ Georg-Brauchle-Ring 60/62 D-80992 München, Germany \\ ${ }^{2}$ Städtisches Klinikum München, Klinik für Neuropsychologie, Englschalkinger Straße 77, \\ Munich 81 925, Germany \\ ${ }^{3}$ Centro de Automática y Robótica, Universidad Politécnica de Madrid, ETSI Industriales, Madrid, Spain
}

marta.bienkiewicz@tum.de, joachim.hermsdörfer@tum.de

Keywords: Apraxia, dynamic cues, biological motion, ecological sounds

Abstract: Technological progress in the area of informatics and human interface platforms create a window of opportunities for the neurorehablitation of patients with motor impairments. The CogWatch project (www.cogwatch.eu) aims to create an intelligent assistance system to improve motor planning and execution in patients with apraxia during their daily activities. Due to the brain damage caused by cardiovascular incident these patients suffer from impairments in the ability to use tools, and to sequence actions during daily tasks (such as making breakfast). Based on the common coding theory (Hommel et al., 2001) and mirror neuron primate research (Rizzolatti et al., 2001) we aim to explore use of cues, which incorporate aspects of biological motion from healthy adults performing everyday tasks requiring tool use and ecological sounds linked to the action goal. We hypothesize that patients with apraxia will benefit from supplementary sensory information relevant to the task, which will reinforce the selection of the appropriate motor plan. Findings from this study determine the type of sensory guidance in the CogWatch interface. Rationale for the experimental design is presented and the relevant literature is discussed.

\section{INTRODUCTION}

Smart prompting technologies play an increasing role in providing daily assistance to people suffering from compromised cognitive functioning (Seelye et al., 2011). The aim of the proposed study is to investigate the application of dynamic cues based on biological motion and ecological sounds to improve daily activities in a group of apraxic stroke survivors. In its simplest form, apraxia can be defined as a loss of the ability to use tools or perform hand gestures, and is typically caused by brain tissue loss in the parietal and frontal lobe areas of the left hemisphere (Goldenberg et al., 2007). Recent research conducted in UK, suggests that approximately $24 \%$ of stroke survivors have persistent signs of apraxia (Bickerton et al., 2012). Another group of patients that have difficulties with daily activities are those with action disorganisation syndrome (ADS). ADS patients typically suffer from bilateral frontal brain damage and have similar deficits to apraxic patients when performing daily activities (Cooper et al., 2005). Increasing the independence of apraxia and ADS patients during everyday tasks (such as making tea, grooming, and eating) is a matter of priority for patients, their caregivers, and clinicians (Hazel, 2012). 


\section{BACKGROUND}

\subsection{Apraxia as a stroke consequence}

Apraxia is a neurological sign of brain damage, behaviourally observed as the inability to perform skilled, well-learned motor acts. Those acts might be transitive (i.e. involving tools or multi-step actions, such as sawing a piece of wood or teeth brushing) or intransitive (e.g., pantomime of gestures and imitation tool use) (Gross et al., 2008). These two different subtypes of apraxia are classified separately - conceptual apraxia and ideomotor apraxia respectively, but might coincide together. An important feature of apraxia is that it is independent from other sensory, or motor problems (such as paresis or spasticity) that might occur as a consequence of stroke damage. Due to apraxia, patients are prone to conceptual, spatial and temporal errors during daily activities that can lead to potential health and safety issues (e.g., grasping the knife by the sharp end, pouring boiling water onto the kitchen desktop). Common errors include problems with sequencing in multistep actions (e.g., action addition, omission, anticipation and perseveration errors) along with conceptual errors (e.g., misuse of objects, object substitution, hesitation, toying and mislocation) (Petreska et al., 2007). The difficulty with the use of objects is a source of frustration for patients, making them rely on caregivers for help in everyday life. This loss of independence compounds the problems associated with stroke, and makes the consequences of apraxia more debilitating (Hanna-Plady et al., 2003).

Although clinicians have established a set of well-developed assessment tools to trigger apraxic behaviour, the underlying mechanisms of error production are still not well understood (Goldenberg et al.,. 1996). The cognitive aspect of apraxia (i.e., the loss of knowledge or memory of how the action is performed) is often accompanied by changes in the kinematic pattern of the movement in the unimpaired hand. In the latter case, features such as grip aperture, time to peak velocity, deceleration phase are pointed out as possible kinematic markers of apraxia (Laimgruber et al. 2005). These difficulties, along with the loss of conceptual knowledge, create a void that could be filled by intelligent assistive technology that could facilitate patients' motor performance during everyday activities.
Finding optimal cues (prospective information) that could be implemented in the assistive system for patients is one of the priorities of the CogWatch project. In this paper we explore and compare static and dynamic cues that could aid the daily functioning of patients. Of particular interest is to validate whether cues can provide information ahead of time to facilitate the motor execution in patients who exhibit impaired kinematic performance. Dynamic cues can incorporate both spatial and temporal aspects of the movement, or as in the case of prompts, provide verbal instruction about the next step of the action. In this study, we will investigate the use of cues that account for both conceptual and kinematic deficits in apraxia. This will be presented in the following section.

\subsection{The aim of the CogWatch project}

The aim of the CogWatch project is to provide an online prompting system that can be implemented in the home setting (Giachristis et al., submitted). This system is comprised of three technological modules: instrumented tools that provide feedback to the system indicating how an object is being manipulated, CogWatch wrist worn device that provides feedback about the errors and prompting instructions to a patient, and a Virtual Task Execution (VTE) screen that provides prospective sensory guidance about the appropriate object and tool action and execution.

Additionally, the feedback system will be based on two types of cues: semantic feedback (i.e., information that the error was made via visual, sensorimotor and auditory channel) and dynamic cues that provide prospective information as to how the next step of action should be performed (based on the motion capture recordings of healthy individuals performing and action with the same objects and task scenario).

\subsection{Prospective cues}

Cues are widely embedded in our everyday environment, for example in traffic lights that signal when it is a safe time to cross a road. The term 'cue' can be defined as external information relevant to the movement execution (Horstink et al., 1993). In general, cues are divided into sources of spatial and temporal information. Gibson (1950) proposed that the environment is built of structured arrays of sensory information that we can perceive through different sensory modalities. Spatial cues can 
provide information about where to aim a movement (e.g., target space on an object) whilst temporal cues can provide information about when to execute the movement (e.g. a metronome that triggers a "move now" response). For example, if the goal of the action is to grasp a moving object, successful interception with the target requires the action to be coupled to the motion path of the object. Object in motion can provide continuous information on both the spatial and temporal dimensions that directly guides our motor planning. By comparison, a stationary object can only provide spatial cues about the current placement of the object without additional temporal information. Using sensory information to guide goal-directed actions relies on the indirect sensorimotor pathways in the basalganglia circuitry that are involved in motor learning and interaction with the environment (Redgrave et al., 2010).

Cues can be presented in all sensory modalities: visual, acoustic, haptic, somatosensory (Sveistrup, 2004), and can be static or dynamic (Amblard et al., 1985). So far, the use of cueing paradigm has been most effectively applied to rehabilitation of motor impairments in Parkinson's disease, such as gait (Nieuwboer et al., 2007), and arm movements in the hemiparesis following stroke (Thaut et al., 2002). Since apraxia is a multifaceted syndrome, an effective cueing method needs to prevent patients from committing both conceptual and dynamic errors during their task performance. We propose that cues based on biological motion recordings of healthy adults performing transitive and intransitive movements are potentially a best fit for further exploration as they have a potential to encapsulate both motor concept and efficient motor programme.

\subsection{Two lines of exploration: cues based on biological motion vs. ecological sounds}

The aim of this study is to verify which cues are best tailored to the needs of patients with apraxia syndrome, based on the plethora of research dedicated to action perception coupling. To do so, we propose two paths of exploration. First, to test the cues based on the biological motion of a healthy adult performing the action (transitive and nontransitive), incorporated in a simulation of a moving avatar on the VTE screen. Second, to test the use of ecological sounds linked to achieving the goal of the action (e.g., the sound of the tooth brushing) alone and incorporated in the animations.

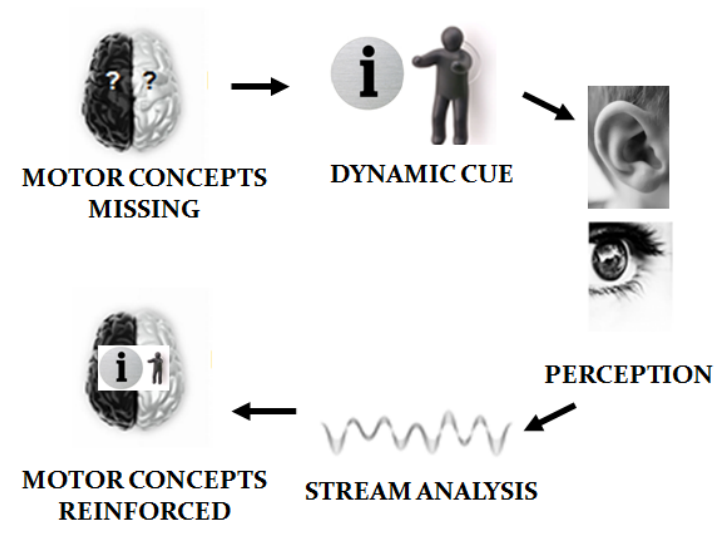

Figure 1: Illustration of the cueing paradigm for apraxia patients and how relevant information about the motor concept could be extracted from the dynamic cues of different sensory modalities. Information perceived (for example via visual channel) is analysed in terms of temporal and spatial features and its relevance (meaning) which feeds into missing motor concepts and movement planning.

\subsubsection{Incorporating biological motion into avatar movement.}

The idea that the observation of another person's movement can activate motor representation stems from the research on primate subjects conducted by the Parmesan group of Rizzolatti (see Rizzolatti \& Craighero, 2004; Prinz, 1997). Researchers have identified a class of neurons, referred in the literature as 'mirror neurons' that are activated when one performs a motor action, and when observing another individual performing this action (primate and human studies). Perception of the action of others not only discharges neurons involved in motor representation, but also consequently facilitates acts that are congruent to the displayed action performance and inhibits actions that are not congruent with the observed motion (Christensen et al., 2011). In primate research, the discharge of mirror neurons was demonstrated to be linked to the availability of the goal of action. That is, transitive actions only (Rizzolatti et al., 2001). In humans, however, the activity of the mirror neuron network is not determined by the goal of action, as intransitive acts also can elicit discharge of those neurons (Jackson et al., 2005; Fadiga et al., 1995, Tanaka et al., 2002). Interestingly, intransitive actions are the first actions that are copied by human newborns (Meltzoff et al., 1977). Gallese and Goldman (1998) 
suggested that the mirror neuron network plays a crucial part in motor learning in humans, as it facilitates the acquisition of motor skills (such as tool use) through imitation. In summary, there is a body of research suggesting that sensory information linked to the action in the environment, is mapped onto the motor representation of this action (Rizzolatti et al., 2001). Usually action observation imposes $3^{\text {rd }}$ person perspective perception (see Figure 2). However, Jackson et al. (2005) have found a subtle difference (in terms of brain activation patterns) between observation and imitation of motor acts in humans, depending on the perspective of the person perceiving a motor event. Their work, based on fMRI investigation, suggests more tight links between $1^{\text {st }}$ person perspective and the sensorimotor system, compared to the $3^{\text {rd }}$ person perspective that requires additional transformation of the visuospatial perspective. In line with their findings, observing an action from a $1^{\text {st }}$ person perspective does not require additional mental operations, and therefore might be better suited to imitation learning. Indeed, limited evidence from clinical apraxia research suggests that patients with apraxia make less motor errors in pantomime when an experimenter is demonstrating the action when seated next to them, rather than vis-à-vis (Jason, 1983).

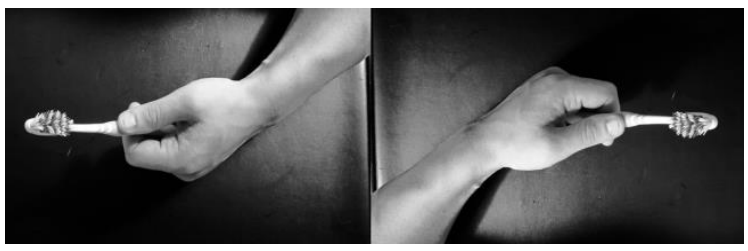

Figure 2: Illustration of the $1^{\text {st }}$ and $3^{\text {rd }}$ person perspective on the example of tooth brushing. The photo on the left illustrates $1^{\text {st }}$ person perspective (left hand), the photo on the right $3^{\text {rd }}$ person view (left hand).

The novel aspect of this study is to use biological motion displays that provide temporal characteristics of the movement that can be incorporated into motor planning (see Figure 1). From the mirror neuron perspective, the observation of an avatar performing an action (e.g., tooth brushing) has the potential to facilitate action performance in apraxia patients. Limited research on the use of cues in apraxia suggests that the addition of somaesthetic cues may improve certain aspects of apraxic movement (de Renzi et al., 1982). The supplementary information prescribed by the cues might promote the selection of an adequate motor program (Hermsdörfer et al., 2006).

\subsubsection{Ecological sounds}

Vision is the most intuitive sensory modality that allows us to interact with the environment and to regulate our movements (Goodale, \& Humphrey, 1998). However, recent scientific evidence suggests that vision, audition, and haptic modalities are partially interchangeable (Zahariev, \& MacKenzie, 2007). Humans are capable of identifying both the size and shape of an object dropped onto a surface using auditory feedback of the event, without any visual information or previous knowledge of the object (Laktos et al., 1997; Grassi, 2005; Houben et al., 2005). The common coding approach suggests that motor representations can be accessed through different sensory modalities, as the sensory representations are shared in the brain (Hommel et al., 2001). Importantly, previously mentioned research on motor neurons, also shows that mirror neurons discharge when the action-related sounds were made available without the action being visible (Kohler et al, 2002, Keysers et al, 2003). Another recent investigation has demonstrated that mirror neurons can respond to newly acquired associations between sounds (not relevant to action) and actions via learning (Ticini et al., 2012). This suggests that the human brain operates on a high-order sensorymotor representation level, which is independent from the afferent input and directed at the goal of actions. In line with ideomotor theory, some authors speculate that action goals are tangled with the expected sensory feedback (Prinz, 1997).

Ecological sounds (i.e. sounds that are linked to the goal of the production, such as the sound of a nail hit by a hammer, a passing helicopter, or a bouncing ball) contain spatiotemporal characteristics that allow humans to successfully interact with the environment. For example, to avoid colliding with a moving object (e.g., passing car) or intercept with the environment (e.g., catch a ball). In addition, ecological sounds have been demonstrated to boost motor performance in Parkinson's disease. Young et al. (2012) used the sound of walking on gravel, with different gait characteristics (e.g., stride amplitude, cadence) to facilitate walking in people with moderate to advanced Parkinson's. In those patients, an 
improvement in gait pattern was observed when their steps were mapped to the sound of walking, delivered via headphones.

In this study, we propose using sounds that are associated with everyday actions - the sound of tooth brushing, sawing and hammering. These cues will be compared to verbal commands, avatar displays in the $1^{\text {st }}$ and $3^{\text {rd }}$ person (see Figure 2), and still pictures.

\subsection{Experimental design}

In the pilot study phase, cues will be validated in a group of five neurologically healthy adults. Further, a group of 10 patients with recognised apraxia features will be tested, along with 10 age-matched controls to create baseline performance for the patient group. Patients will be recruited from the Klinik für Neuropsychologie in Städtisches Klinikum München (STKM), Germany. Ethical approval was granted for the study by a local committee.

Control and patient groups will be tested under three conditions:
A. Actual action execution
B. Pantomime with action object visible
C. Pantomime with action object not visible

Three daily tasks will be introduced:

- Tooth brushing

- Sawing a piece of wood

- Hammering

The set of cues will comprise of:

$\begin{array}{ll}\text { I. } & \text { No cues, instruction of the task given } \\ \text { before the task starts } \\ \text { II. } & \text { Verbal prompts, step by step } \\ \text { III. } & \text { Simulation 1st perspective } \\ \text { IV. } & \text { Simulation 3rd perspective } \\ \text { V. } & \text { Simulation 1st perspective+sounds } \\ \text { VI. } & \text { Simulation 3rd perspective+sounds } \\ \text { VII. } & \text { Sounds only } \\ \text { VIII. } & \text { Still pictures sequence } \\ \text { IX. } & \text { Still pictures sequence plus sounds }\end{array}$

Participants will perform each of the tasks under three conditions with the set of IX cueing blocks for each condition. Motor performance will be recorded using high frequency video cameras and an ultrasonic motion capture system (Zebris). Pantomime and tool use will be assessed using the Goldenberg \& Hagmann (1998) 2 point scale. The following kinematic variables noted in the literature as motor features of apraxia (Laimgruber et al., 2005) will be analysed: movement time (MT), peak velocity (PV), deceleration phase (DP) and grip aperture (GA). In addition, errors will be categorized according to the error classification proposed by Schwartz et al. (1995). To observe how presentation of cues can moderate motor planning in patients, error corrections will be subdivided into two categories: pre-error correction (e.g., hesitation before performing a movement in a wrong spatial position) and post-error correction (e.g., changing the spatial position after the movement proved to be ineffective). Number of errors committed and kinematic features of the movement will be compared across conditions for each patient and groups between patients and age-matched controls.

\section{RESEARCH AIMS}

The purpose of this research is to explore how patients with apraxia can benefit from the availability of artificial environmental sensory information that can be harnessed for motor planning. The rationale behind the study is based on the assumption that patients will be able to extract this information to aid their own cognitive and kinematic deficits of tool use and gesture production. The critical question is to define which cues have the greatest potential to be utilised by patients to prospectively guide their movements and effectively be implemented in the CogWatch interface.

In addition, the study aims to explore how perception of biological motion displays moderate behaviour in apraxia patients, depending on the perspective of perception $\left(1^{\text {st }}\right.$ person versus $3^{\text {rd }}$ person). To the best knowledge of the authors, this study is the first to address this issue in individuals with apraxia. We hypothesize that task performance will improve in terms of the decreased number of conceptual and motor errors committed when dynamic cues are made available (the ones based on the biological motion and ecological sounds), in comparison to task performance when no cues are available, or they are static and do not contain biological movement patterns. 


\section{CONCLUSIONS}

The work on this project is still ongoing and requires detailed experimentation with selected stroke survivors that show persistent signs of apraxia. On the basis of the data analysis from the proposed study, the most effective method of cueing action use and pantomime will be implemented in the CogWatch interface.

The current development of the CogWatch system aims to provide a user-friendly, home-based solution for stroke survivors that will improve their degree of independence during activities of daily living. In addition, the user-experience will be enhanced by providing a customised interface for each patient. The interface will be tailored to the personal preferences and requirements, to increase the comfort of interaction, ecological validity and efficacy of the system. For example, the avatar used in the simulation will be personalised to resemble the patient and his home environment. The cueing method will also be adjusted to the capabilities of the patients, for example, whether the person is able to move both arms or just one (hemiparesis). This non-immersive system will allow the transfer of information that is necessary to successfully accomplish daily activities such as preparation of food.

Importantly, the CogWatch system is designed to be an entirely non-invasive and affordable solution for the majority of patients. The technology implemented in the project so far (KinectTM system and VTE monitor) is relatively low cost and is under continuous development by the manufacturers. Furthermore, it offers flexibility in adjusting and updating the interface to the changing needs of patients (for example, partial recovery). Finally, the CogWatch system is targeted at home-based rehabilitation of the patients in a chronic phase of the disease, when often they do not have further access to rehabilitation from their medical providers. The CogWatch system will deliver affordable and easy access to therapeutic intervention at patients' homes, which is the most comfortable and natural setting, without a need for supervision.

An additional benefit for the patient is that the Cogwatch system will be able to provide an online and objective assessment of the patient's progress that can partially replace the clinic visits (Giachristis, \& Randall; submitted). In sum, the CogWatch system will be driven by readily available and cost efficient technology that can be customised to patient requirements in order to heighten userfriendliness.

\section{ACKNOWLEDGEMENTS}

This work was funded by the EU STREP Project CogWatch (FP7-ICT- 288912).

\section{REFERENCES}

Amblard, B., Cremieux, J., Marchand, A. R., \& Carblanc, A. (1985). Lateral orientation and stabilization of human stance: Static versus dynamic visual cues. Experimental Brain Research, 61(1), 21-37.

Bickerton, W., Riddoch, J., Samson, D., Balani,A., Mistry, B., \& Humphreys, G., (2012). Systematic assessment of apraxia and functional predictions from the Birmingham Cognitive Screen, Journal of neurology, neurosurgery \& psychiatry with practical neurology, 83 (5), 513-52.

Christensen, A., Ilg, W., \& Giese, M. A. (2011). Spatiotemporal tuning of the facilitation of biological motion perception by concurrent motor execution. The Journal of Neuroscience, 31(9), 3493-3499.

Cooper, R. P.; Schwartz, M.; Yule, P. \& Shallice, T. (2005). The simulation of action disorganisation in complex activities of daily living. Cognitive Neuropsychology 22 (8) 959-1004.

De Renzi, E., Faglioni, P., \& Sorgato, P. (1982) Modality specific and supramodal mechanisms of apraxia. Brain, 105, 301-312.

Fadiga, L., Fogassi, L., Pavesi, G., \& Rizzolatti G. (1995) Motor facilitation during action observation: a magnetic stimulation study, Journal of Neurophysiology, 73, 2608-2611.

Gallese,V., \& Goldman, A., (1998). Mirror neurons and the simulation theory of mind-reading, Trends in Cognitive Sciences, 2(12), 493-501.

Giachristis, C., \& Randall, G. (submitted) CogWatch: Cognitive Rehabilitation for Apraxia and Action Disorganization Syndrome Patients. $6^{\text {th }}$ International Conference of Health Informatics.

Gibson, J. J. (1950). Perception of the visual world. Boston: Houghton Mifflin. 
Goldenberg, G., Hermsdörfer, J., Glindemann, R., Rorden, C., \& Karnath, H. O. (2007). Pantomime of tool use depends on integrity of left inferior frontal cortex. Cerebral Cortex, 17, 2769-2776.

Goldenberg, G., \& Hagmann, S. (1998) Therapy of activities of daily living in patients with apraxia. Neuropsychological Rehabilitation, 8(2), 123-41.

Goldenberg, G., Hermsdörfer, J., \& Spatt, J. (1996). Ideomotor apraxia and cerebral dominance for motor control. Cognitive Brain Research, 3, 95-100.

Goodale, M. A., \& Keith Humphrey, G. (1998). The objects of action and perception. Cognition, 67(1-2), 181-207.

Grassi, M. (2005). Do we hear size or sound? balls dropped on plates. Attention, Perception, \& Psychophysics, 67(2), 274-284.

Gross, R., \& Grossman, M. (2008). Update on apraxia. Current neurology and neuroscience reports, 8 (6), 490-496.

Hazell, A. (2012) CogWatch - Cognitive Rehabilitation of Apraxia and Action Disorganisation Syndrome: Assessing the requirements of Healthcare Professionals, Stroke Survivors and Carers. UK Stroke Forum Conference 2012 Proceedings.

Hanna-Pladdy, B., Heilman, K.M., \& Foundas, A.L. (2003) Ecological implications of ideomotor apraxia evidence from physical activities of daily living. Neurology, 60, 487-490.

Hermsdörfer, J., Hentze, S., \& Goldenberg, G. (2006) Spatial and kinematic features of apraxic movement depend on the mode of execution. Neuropsychologia, $44,1642-1652$.

Hommel, B., Müsseler, J., Aschersleben, G., \& Prinz, W. (2001). The theory of event coding (TEC): A framework for perception and action planning. Behavioral and Brain Sciences, 24(05), 849.

Horstink, M., De Swart, B., Wolters, E., \& Berger, H. (1993). Paradoxical behavior in Parkinson's disease. In: E. C Wolters, \& P. Scheltens (Eds). Mental dysfunction in Parkinson's disease; proceedings of the European congress on mental dysfunction in Parkinson's disease. Amsterdam: Vrije Universiteit, 1993. Proceedings of the European Congress on Mental Dysfunction in Parkinson's Disease.
Houben, M., Kohlrausch, A., \& Hermes, D. (2005). The contribution of spectral and temporal cues to the auditory perception of size and speed of rolling balls. Acta Acustica United with Acustica, 91(6), 1007.

Jackson, P., Meltzoff, A., \& Decety, J. (2006) Neural circuits involved in imitation and perspective-taking. Neuroimage, 15 (31), 429-439.

Jason, G. W. (1983). Hemispheric asymmetries in motor function: Left hemisphere specialization for memory but not performance. Neuropsychologia, 21 (1), 35-45.

Keysers, C., Kohler, E., Umiltà, M.A., Nanetti, L., Fogassi, L., \& Gallese, V. (2003). Audiovisual mirror neurons and action recognition, Experimental Brain Research, 153 (4), 628-636.

Kohler, E., Keysers, C., Umilta, M., Fogassi, L., Gallese, V., \& Rizzolatti, G. (2002). Hearing sounds, understanding actions: action representation in mirror neurons. Science, 297, 846-848.

Laimgruber, K., Goldenberg, G., \& Hermsdörfer, J. (2005) Manual and hemispheric asymmetries in the execution of actual and pantomimed prehension, Neuropsychologia, 43(5), 682-692.

Lakatos, S., McAdams, S., \& Caussé, R. (1997). The representation of auditory source characteristics: Simple geometric form. Attention, Perception, \& Psychophysics, 59(8), 1180-1190.

Meltzoff, A. N., \& Moore, K. (1977) Imitation of Facial and Manual Gestures by Human Neonates, Science 198 (4312), 75-78

Nieuwboer, A., Kwakkel, G., Rochester, L., Jones, D., van Wegen, E., Willems, A. M. et al. (2007). Cueing training in the home improves gait-related mobility in Parkinson's disease: The RESCUE trial. Journal of Neurology, Neurosurgery \& Psychiatry, 78(2), 134140 .

Petreska, B., Adriani, M., Blanke, O. \& Billard, A. (2007) Apraxia: a review. In C. von Hofsten (Ed.). From Action to Cognition. Progress in Brain Research. Elsevier. Amsterdam. Vol. 164, pp. 61-83

Prinz, W. (1997). Perception and action planning. European Journal of Cognitive Psychology, 9 (2), 129-154. 
Redgrave, P., Rodriguez, M., Smith, Y., Rodriguez-Oroz, M., Lehericy, S., Bergman, H., et al. (2010). Goaldirected and habitual control in the basal ganglia: Implications for Parkinson's disease. Nature Reviews. Neuroscience, 11(11), 760-772.

Rizzolatti, G., Fogassi, L., \& Gallese, V. (2001) Neurophysiological mechanisms underlying the understanding and imitation of action. Nature Reviews. Neuroscience, 2(9), 661-670.

Rizzolatti, G., \& Craighero, L. (2004). The mirror-neuron system. Annual Review of Neuroscience, 27(1), 169192.

Schwartz, M. F., Montgomery, M. W., Fitzpatrickdesalme, E. J., Ochipa, C., Coslett, H. B., \& Mayer, N. H. (1995). Analysis of a disorder of everyday action. Cognitive Neuropsychology, 12, 863-892.

Seelye, A., Schmitter-Edgecombe, M., Das, B., \& Cook, D. (2011). Application of Cognitive Rehabilitation Theory to the Development of Smart Prompting Technologies, Biomedical Engineering, IEEE Reviews, 99, pp.1, 0.

Sveistrup, H. (2004). Motor rehabilitation using virtual reality. Journal of NeuroEngineering and Rehabilitation, l(1), 10.

Tanaka, S., \& Inui, T. (2002) Cortical involvement for action imitation of hand/arm postures versus finger configuration : An fMRI study, NeuroReport 13, 1599-1602.

Thaut, M. H., Kenyon, G. P., Hurt, C. P., McIntosh, G. C., \& Hoemberg, V. (2002). Kinematic optimization of spatiotemporal patterns in paretic arm training with stroke patients. Neuropsychologia, 40(7), 1073-1081.

Ticini, L.F., Schutz-Bosbach, S., Weiss, C., Casile, A., \& Waszak, F. (2012). When sounds become actions: higher-order representation of newly learned action sounds in the human motor system. Journal of Cognitive Neuroscience, 24(2), 464-474.

Young, W.R., Rodger, M.W.M., \& Craig, C.M. (2012) Using ecological event-based acoustic guides to cue gait in Parkinson's disease patients [abstract]. Movement Disorders, 27 Suppl $1: 119$

Zahariev, M., \& MacKenzie, C. (2007). Grasping at 'thin air': Multimodal contact cues for reaching and grasping. Experimental Brain Research, 180(1), 6984. 